\title{
Technical Considerations for Improving Near-field Enhancement Optical Microscopy
}

Erik J. Sánchez ${ }^{1}$, Derek B. Nowak ${ }^{1}$ Jeff J. Doughty ${ }^{1}$, A.J. Lawrence ${ }^{1}$, Amanda Delzer ${ }^{2}$, Abraham Neben $^{3}$, Cliff Kim ${ }^{4}$.

1. Portland State University, Dept. Physics, Portland, OR, USA

2. Lewis and Clark College, Physics Dept., Portland, Oregon, USA

3. University of Chicago, Dept. of Physics, Chicago, IL, USA

4. Syracuse University, L.C. Smith College of Engineering and Computer Science, Syracuse, NY, USA

We have developed a nonlinear imaging system that employs two-photon excitation incident on probe tips in order to achieve super-resolution imaging. The microscope demonstrates the ability to image topographical, optical, and electronic state information for single-molecule identification. The system has a modular design which allows simple modifications with off the shelf parts.

Tip enhanced near-field optical microscope probes are used to image spectroscopic samples with special resolutions below the diffraction limit [1]. Transmission electron microscopes (TEM) play a major role in material development in order to elucidate structural information on a nanometer scale. However large samples and electron conduction experiments within a TEM can be very challenging. With spectroscopic techniques such as Raman or Fluorescence one also has the ability to gain information on structure, but typically on the level of the diffraction limit $(\lambda / 2)$. We developed a nonlinear tip enhanced method of utilizing field enhancement in order to image on the levels far below the diffraction limit [2]. Many factors must be matched perfectly to achieve a high level of field enhancement; one particular important aspect of the field enhancement process involves a well designed probe tip. The proper fabrication of this tip requires the use of a focused ion beam (FIB) system as well as a finite difference time domain (FDTD) modeling.
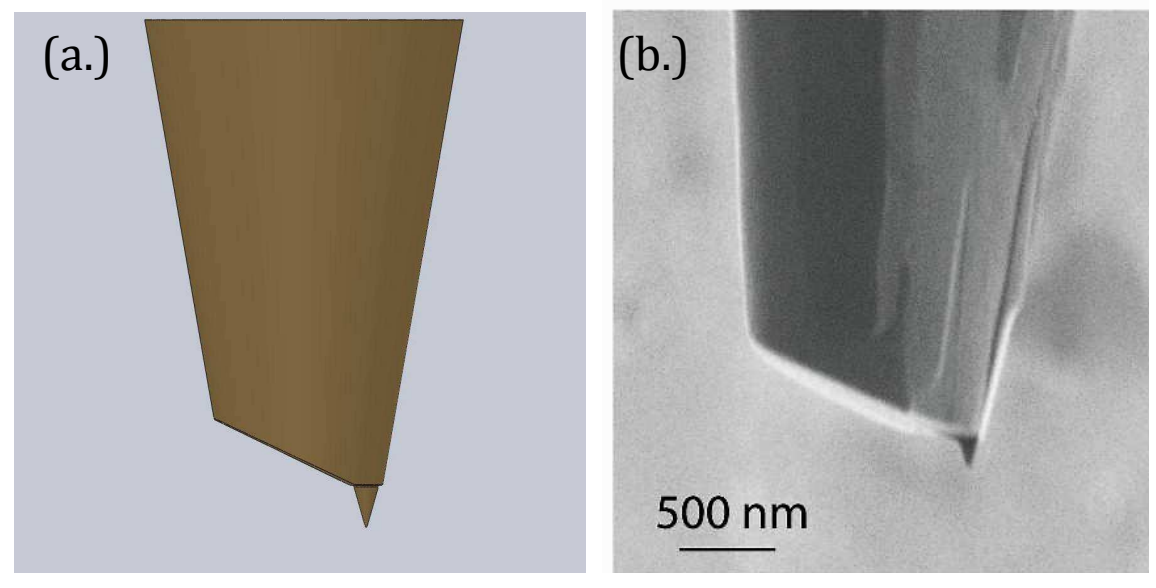

FIG 1: (a.) Artist 3D rendering of the probe tip design.(b.) Actual fabrication of the tip using a dual-beam focused ion beam (FIB).

Many groups have discussed the use of laser-illuminated metal tips for near-field imaging. Most of the work involved the scattering of light of a metal probe. The probe perturbs the fields at the sample; this perturbation can be detected in the far-field as a signal of the same frequency as the incident light. Little is known concerning the contrast in the signals and spectroscopic signals cannot be probed. The process proves to be dominated by the topographic information rather than 
the spectroscopic information. Our system detects the spectroscopic signal from the sample only and not the excitation field. The field is incident upon a metallic probe tip capable of supporting a Plasmon. The probe tip tends to have an asymmetric design associated with it. This topic will be discussed.

Manufacture of an asymmetrical tip design with a focused ion beam microscope is outlined. FDTD modeling of an asymmetrical tip design is presented. A discussion of imaging mechanisms by a comparison of near-field optical and topographical sheer force images with tip design and quenching mechanisms is also discussed.

The near-field multi-photon imaging system based on a mode-locked Ti:Sapphire as well as a CW diode laser. The reason for imaging in a multi-photon mode is based on the versatility of filter selection, use of one wavelength of most visibly excited fluorophores, depth of penetration in the $Z$ direction, and better signal-to-noise contrast due to the non-linear nature of multi-photon excitation. Another advantage of technique with our design involves no requirement of photo beaching of the sample in order to achieve this resolution. The NSF project involved the fabrication of a fluorescence near-field imaging system using a CW diode laser as a replacement for Ti:Sapphire laser for researches more concerned with spatial information than time dynamics as well as those on a restricted budget..

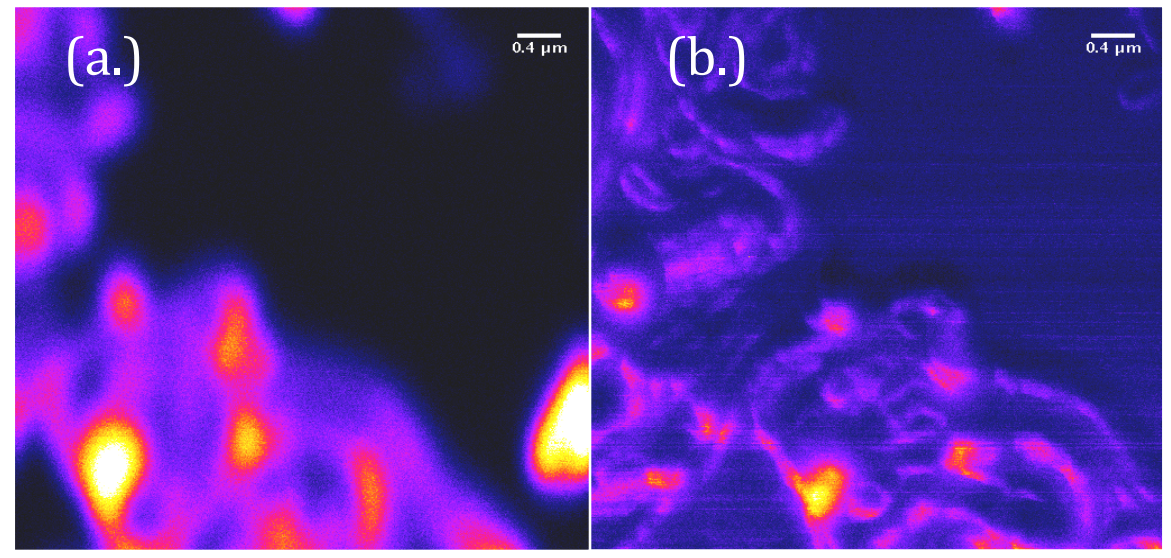

FIG 2: (a.) Far-field multi-photon diffraction limited mapping of J-aggregates with an excitation level of $35 \mu \mathrm{W}$, (b.) a near-field tip-enhanced image of same area using a CW $834 \mathrm{~nm}$ diode laser at $1.3 \mathrm{~mW}$. The resolution is on the order of tens of nanometers.

Figure 2 shows the latest results form our microscope base system so far. The near-field effects of increasing the resolution can be easily seen in figure $2 \mathrm{~b}$ when a probe tip is lowered into the light field.

\section{References:}

[1] E. J. Sánchez, L. Novotny, X.S. Xie, Phys. Rev. Lett. 82, 20 1999, 4014.

[2] Hartschuh, Achim, et al., Phys. Rev. Lett. 90, 9, March 7, 2003, 095503-1.

[3] NSF awards DBI-0500812, ECCS-NSF-0520891, NSF-MRI-\#722660, DMR-NSF-REU\#0649280, DMR-NSF-REU-069280 and NSF-DMR-REU\#1004737, ONAMI/ONR awards (\#N00014-07-1-0457), (\#N00014-08-1-1237), and (\#N00014-10-1-0082)

[4] www.ansom.physics.pdx.edu for project information. 